\title{
¿CUÁNDO UN CONTENIDO ACADÉMICO TIENE SIGNIFICADO PARA EL ALUMNO? IMPLICACIONES DIDÁCTICAS
}

\author{
JIMÉNEZ GÓMEZ, E. ' y MARÍN MARTÍNEZ, N. ${ }^{2}$ \\ t Departamento de Didáctica de las Ciencias Experimentales. Universidad de Murcia. \\ 2 Departamento de Didáctica. Universidad de Almería.
}

\begin{abstract}
SUMMARY
The importance of identifying the prior ideas of students on a given content is based, among other reasons, on the need to accommodate the teaching processes to the cognitive peculiarities of the pupils. However, individuals tend to develop ideas or conceptions only for some academic purpose, that is to say for those that are meaningful to them. Hence the need to establish some observable criterion that permits classification of the content teaching purpose by their degree of meaning level, whose science education interest resides in the utilization of accepted teaching techniques with the said meaning level.
\end{abstract}

\section{INTRODUCCIÓN}

En la década de los años setenta y, especialmente, en los ochenta se ha tenido una gran preocupación por descubrir las concepciones que poseen los alumnos acerca de determinados fenómenos físico-naturales, y hacerlas progresar, a través del proceso de enseñanza, hacia las que comparte la comunidad cientifica. Esto ha originado una línea de investigación en la didáctica de las ciencias experimentales que ha aportado un importante catálogo de dichas concepciones (Carmichael et al., 1990; Confrey, 1990).

En los catálogos anteriores se identifican y describen las concepciones de los alumnos para la casi totalidad de conceptos básicos de ciencias: fuerza, energía, reflexión de la luz, campos magnéticos, reacciones químicas, estructura de la materia, digestión, átomo, ion, etc. Sin cmbargo, el nivel o grado de significación que poseen los diferentes contenidos para el alumno no son especial- mente analizados, a pesar de tener una gran importancia a la hora de llevar a cabo los diseños de enseñanza y la construcción de materiales didácticos.

Existen conceptos como velocidad, peso, equilibrio físico, etc. para los cuales el sujeto tiene esquemas fruto de sus interacciones con su medio físico y social; sin embargo, para otros de mayor nivel de abstracción o más alejados de su medio cotidiano, como son ion, diferencia de potencial eléctrico, mol, fotosíntesis, energía química, etc., será difícil encontrar esquemas en el sujeto que le permitan asociar significados, más o menos adecuados, a dichos conceptos. Por tanto, no parece lógico que se puedan investigar y menos aún describir las concepciones de los alumnos para casi todos los contenidos académicos. De aquí, la necesidad de establecer criterios que permitan diferenciar contenidos objeto de enseñanza por su nivel de significación. 
En este trabajo se establecen algunos criterios mínimos, fundamentados en aspectos cognoscitivos del alumno, que permiten una aproximación al estudio y ponderación del grado de signifícación que un determinado contenido objeto de enseñanza posee para un alumno. Una vez determinado dicho grado de significación se analizan sus implicaciones en la enseñanza y se presentan algunas sugerencias metodológicas para la detección de las concepciones de los alumnos.

\section{EL CONTENIDO COGNOSCITIVO DEL SUJETO ESTÁ PRINCIPALMENTE RELACIONADO CON IOS TIPOS DE INTERACCIONES QUE LLEVA A CABO CON SU MEDIO NATURAL Y SOCIAL}

La estructura cognoscitiva se genera por la interacción del sujeto con su medio natural y social, siendo Ias interacciones mecánicas las que inducen en el sujeto un mayor número de concepciones de forma espontánea (Piaget y García, 1973), y donde el componente social tiene posiblemente una menor influencia, lo que explica que un buen número de dichas concepciones están ligadas a fenómenos tales como: sopesar, tirar, empujar, etc. (Hierrezuelo y Montero, 88, pp. 68-72).

En fenomenologías físicas más alejadas de la interacción del sujeto con su medio, como pueden ser la eléctrica, térmica, luminosa, etc., el sujeto desarrolla concepciones más de origen analógico o social que espontáneo (Pozo et al., 1991).

Con frecuencia, cuando el contenido a investigar es poco significativo para los alumnos, el educador o investigador suele describir las concepciones por categorías que denotan el desconocimiento que presenta el alumno sobre dicha materia o por los errores conceptuales que comete en sus explicaciones (Acevedo, 1990; Heller y Finley, 1992; Closset, 1989; Gabell et al., 1987). Sin embargo, cuando un alumno realiza explicaciones sobre sucesos mecánicos, las concepciones suelen ser más fáciles de identificar y describir. Ello es debido a que las operaciones que se ponen en juego en dichas explicaciones provienen de acciones o coordinación de acciones impuestas desde los comienzos del desarrollo por ta estructura de nuestro organismo (Piaget y García, 1973, pp. 18-19), algo que no sucede, al menos con análoga intensidad, en otras fenomenologías.

El único modo que el sujeto tiene de conocer o dar significado a cualquier dato externo es mediante la utilización de algún esquema de asimilación (Piaget, 1977), pero si éste no existiese o fuese inadecuado, dicho dato no pasaría de ser una percepción primaria imposible de ser asimilada y, si se captara, se haría de forma distorsionada o sesgada.

La dificultad de operar con este tipo de datos que sufren una asimilación distorsionada puede lievar con frecuen- cia al sujeto a dar respuestas de compromiso que no denotan elementos cognoscitivos relacionados con 10 que se pregunta, ya que no ha habido asimilación, ésta ha sido inadecuada o ha sido inducida socialmente.

A fin de trabajar con situaciones físicas cuyos datos puedan ser asimilados (datos significativos), al menos en parte, por el sujeto, habrá que comenzar realizando una investigación previa para seleccionar situaciones que sean significativas para él. De entrada, las situaciones mecánicas donde se ponen en juego acciones como empujar, tirar, presionar, equilibrar, suspender, etc. son las más significativas, por las razones dadas.

\section{CRITERIO DE SIGNIFICACIÓN}

Para establecer un criterio de significación se van a diferenciar dos planos observables, el de la tarea compuesta por las situaciones físicas y cuestiones asociadas a éstas y el de las respuestas del sujeto al enfrentarse con ellas. Dentro de este último plano, las novedades, ausencias o distorsiones contenidas en Ias respuestas del sujeto, respecto al aspecto perceptivo con que se presenta la tarea, deben permitir analizar en qué medida el sujeto ha hecho intervenir su bagaje cognoscitivo para elaborar dichas respuestas.

Se puede considerar que una tarea es relevante o significativa cuando el sujeto posee uno o varios esquemas, mediante los cuales se ponen en juego procedimientos de asimilación y acomodación, de forma que éste puede dar significado o interpretar los datos empíricos y así ofrecer respuestas donde, de algún modo, dichos datos sufren algún tipo de transformación (Piaget, 1981, p. 89, p. 104). Por ello, tomaremos como criterio observable de significación el siguiente: si en una respuesta del alumno encontramos nociones, relaciones novedosas $y$, en general, transformaciones no dadas en los datos presentados en la tarea, se podrá decir que la pregunta y la situación física presentada es significativa y, por tanto, el contenido implicado también lo será.

Consecuentemente, un contenido tendrá un grado de significación bajo o nulo para un alumno cuando en sus respuestas utiliza sólo elementos tomados del aspecto perceptivo de la tarea («describe lo que ve»), evoca aspectos irrelevantes, establece analogías inadectladas o expresa su desconocimiento sobre la situación.

Todo lo anterior implica, a su vez, que no podemos saber previamente si una situación física, en la que se ha puesto en juego un determinado contenido, es más o menos significativa hasta que no estamos en presencia de la respuesta del sujeto.

En este sentido, en el diseño de un cuestionario se debería llevar a cabo un proceso de aproximaciones sucesivas entre los esquemas del entrevistador y los del entrevistado. Así, es lógico pensar que en un primer cuestionario deben predominar los esquemas del inves- 
tigador y, sólo evaluando las respuestas del alumno sobre la base del criterio de significación establecido, se podría transformar para acercarlo al alumno y, por tanto, intentar que dicho cuestionario fuese lo más significativo posible.

En el proceso de acercamiento progresivo del cuestionario al alumno se pueden dar varios casos, al evaluar sus respuestas mediante el criterio de significacion:

1) Que las respuestas sean significativas, en cuyo caso la tarea también lo será, así como el concepto a investigar.

2) Que éstas no sean significativas. Aquí puede ocurrir que la tarea seleccionada no sea significativa o que el concepto no lo sea; en este último caso, el dilema se resuclve sustituyendo la tarea presentada.

Un concepto puede ser significativo, según el criterio propuesto, para un alumno de una determinada edad y estadio cognitivo y no serlo para otro sujeto. De ahí la necesidad de realizar estudios evolutivos sobre dicho concepto y deducir, a partir de dichos estudios, cuando un concepto tiene significado para un alumno o grupo de ellos.

\section{ESTUDIOS SOBRE, EL GRADO DE SIGNIFICACIÓN DE ALGUNOS CONTENIDOS ACADEMICOS}

A partir de la aplicación del criterio de significación se va a proceder a realizar un análisis práctico para evaluar el grado de significación de algunos conceptos académicos.

\section{Ejemplo de tarea poco significativa cuando puede serlo el comtenido}

Watts y Zylberstajn (1981), para identificar las nociones que tenían los alumnos de 14 años sobre el concepto de fuerza (interacción), utilizaron la siguiente tarea: Un astronauta en la luna suelta un objeto, a continuación presentan cinco dibujos donde se proponen en cada uno Ia posible fuerza que actúa sobre dicho objeto (hacia arriba, abajo, derecha e izquierda y sin que actúe ninguna fuerza), solicitándose al encuestado que seleccione el caso que considere más acertado, y después que dé una explicación. Aquí, los alumnos sólo pueden dar cinco posibles respuestas, es decir, las que los autores han propuesto; por lo que existe una primacía del punto de vista del investigador sobre lo que pudiera pensar espontánéamente el sujeto.

En esta tarea, tal y como está establecida, no se puede aplicar el criterio de significación, pues las preguntas realizadas invalidan la posibilidad de observar si el contenido y la situación física tienen significado para el alumno.

En el caso de haberse realizado una pregunta abierta, el astronauta en Ia Iuna tampoco parece ser Ia situación física más adecuada para investigar las ideas de los alumnos sobre el concepto de fuerza, puesto que el alumno carece de experiencia personal en dicha situación.

\section{Ejemplos de tareas que permiten establecer el grado de significación de un concepto}

Si se admite que el contenido cognoscitivo del alumno está determinado en buena medida por el tipo de interacción que lleva a cabo con el medio, posiblemente el alumno habrá generado esquemas relativos a conceptos académicos sobre equilibrio mecánico, y esto es así porque:

1) Desde su nacimiento, el niño está sujeto a las fuerzas de la gravedad. Andar supone un mantenimiento continuo de situaciones de equilibración de su propio cuerpo. Aprende a mantener este equilibrio corporal en las más variadas situaciones: sujetando otros objetos de diferentes pesos, en superficies inclinadas o irregulares...

2) El niño gusta de crear situaciones de equilibrio jugando con gran diversidad de objetos, colocándolos unos sobre otros, lo que le permite, en algunos casos, comprobar a su modo la importancia de la vertical en la consecución de equilibrios difíciles.

3) Ha vivido multitud de situaciones donde es necesario conseguir equilibrar determinados objetos a fin de conservar su integridad, de forma que un fracaso de su acción en algunos casos deriva en una ruptura del objeto, por lo cual, incluso, ha podido sufrir un castigo.

4) Muchas diversiones feriales fundamentan su oferta en un intento de desequilibrar a los individuos que intervienen o creando cierta sensación de ingravidez (generalmente suelen poner en juego fuerzas inerciales).

5) Bastantes números que se representan en espectáculos circenses basan su estrategia en la consecución de equilibrios no usuales y difíciles.

Elegido un contenido, que inicialmente parece poseer un alto grado de significación para el alumno, se plantea el problema de diseñar tareas que permitan contrastar el grado de significación de conceptos relacionados con el equilibrio mecánico (verticalidad, centro de masas, dirección del peso, etc.), pues pođría ocurrir que un mal điseño invalidara o impidiera tal contrastactón.

En el diseño de las tareas se deben tener en cuenta algunas consideraciones;

1. Puesto que los aspectos figurativos de las situaciones físicas determinan las respuestas del sujeto (Marín, 1995), es necesario presentar diferentes situaciones donde cambien dichos aspectos para ver su influencia.

Por ejemplo, se han diseñado las situaciones físicas de la figura 1 para conocer los esquemas explicativos (Jiménez Gómez, Solano y Marín, 1994) que tienen los alum- 
nos sobre los conceptos de verticalidad, centro de masa, dirección de los pesos y equilibrio entre fuerzas. Con ello se quiere hacer variar los distintos factores que intervienen en la tarea (forma del alambre y de la plastilina, peso de ésta, etc.) y conseguir poner en juego en toda su extensión los esquemas utílizados por el sujeto al solicitar previsiones, explicaciones, rectificaciones, nuevas explicaciones después de asimilar nuevos datos, etc. (Marín, 1995).

La variabilidad de los distintos factores que intervienen en la tarea permite evaluar el grado de flexibilidad y estabilidad de las respuestas, Io que permite discriminar respuestas de compromiso o dadas in situ (McClelland, 1984) de aquellas otras que puedan ser un buen reflejo de los esquemas espontáneos que el sujeto ha generado.

2. Procurar condicionar las respuestas de los alumnos lo menos posible, evitando la infiuencia del punto de vista del investigador al no hacer girar las cuestiones sobre los significantes académicos (no se ha preguntado en ningún momento sobre el centro de masas, la vertical, la dirección de los pesos y el equilibrio entre fuerzas). Para ello, se puede pedir a un alumno, durante la entrevista individual, que prevea en qué posición quedarán una serie de objetos sencillos como los de la figura 1 cuando se cuelgan del gancho. Además de las descripciones verbales se les pide también que dibujen dichas posiciones.

Figura 1

Objetos utilizados para ser colgados (ele, percha, aro y zapato).

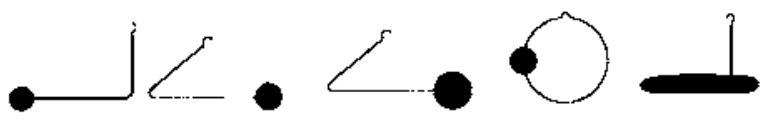

Una vez que los alumnos han realizado los dibujos, se le puede pedir que den explicaciones sobre la posición prevista. Agotadas las respuestas de los entrevistados, se cuelgan los objetos y a cada uno de ellos se les pregunta si aprecian diferencias entre su previsión gráfica y la posición adoptada en el experimento, así como nuevas explicaciones sobre la posición especial que tomaba la bola para cada uno de los objetos.

Se ha realizado un acercamiento al entrevistado mediante cuestiones sobre situaciones físicas donde se han puesto en juego de forma indirecta los contenidos académicos; de este modo, al solicitar explicaciones o previsiones, la espontaneidad del entrevistado se verá menos mediatizada. Después corresponde al investigador entrever las ideas de los alumnos que ocasionalmente serían utilizadas como esquemas para dar significado al contenido objeto de enseñanza.

Pueden presentarse al sujeto nuevas situaciones relacionadas con el equilibrio en las cuales subyace análogo contenido académico (Fig. 2). Se puede apreciar que, con un objeto distinto y una situación de equilibrio diferente, se requiere el mismo procedimiento para responder satisfactoriamente: trazando la vertical desde el gancho o desde la parte más saliente del trozo de plastilina que está unido a unos ganchos que, a su vez, están sustentados por un rectángulo de alambre. Para este caso tos conceptos implicados son los de verticalidad, centro de masas, dirección del peso, equilibrio entre fuerzas y el de plano de sustentación.

Figura 2

Estructura de alambre para sostenet un trozo de plastilina («micron).

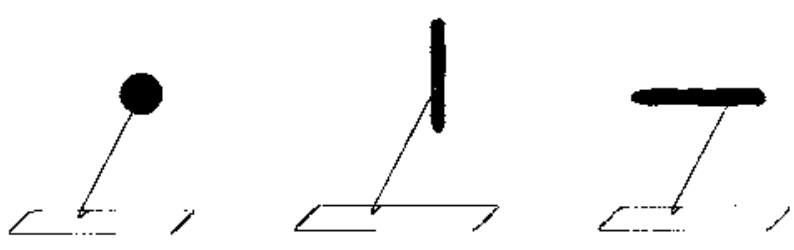

Algunas de las respuestas obtenidas y adecuadamente categorizadas para las preguntas realizadas sobre las situaciones físicas de la figura l son las siguientes:

1) La posición original de los objetos a colgar se deja sin transformar. Antes del experimento, no se hace referencia al peso o no se aprecia en su comportamiento cierta capacidad transformadora. Después de la experimentación se distinguen mal las notables diferencias entre la posición de los objetos dibujados y la observada. La bola de los objetos se deja en un «abajo» no precisado.

a) $\mathrm{Ni}$ antes ni después de la experimentación hacen referencia al peso: Van, Her, Lid, Lor y Pck (iniciales de los sujetos entrevistados).

b) La propia observación lleva a tomar conciencia, por primera vez, de que el objeto se va para abajo debido al peso: Nic y Ram.

2) Se realizan leves transformaciones sobre la posición original de los objetos. La acción del peso es tal que puede ser contrarrestada por la forma. Ahora bien, después de la experimentación, las reacciones son semejantes a las dadas en I: Ner, Lil y Cis.

3) La capacidad transformadora del peso es tál que el sujeto tiene delimitada una zona debajo del gancho, no precisada por la vertical, aunque la forma de algunos objetos impide que lleguen a ella. Las reacciones de los sujetos ante la observación de los objetos colgados permiten distinguir dos subcategorfas:

a) Las diferencias entre posiciones dibujadas y observadas son mejor apreciadas que en la categoría 1 y, en momentos esporádicos y por sugerencias de la observación, se aprecia la relación vertical entre gancho y bola, pero no se aplica sistemáticamente: Fan, Cis, Rom, Ser y Pat.

b) Las diferencias se aprecian sin dificultad y una vez descubierta la vertical se aplica siempre, excepto para el caso del aro: Rev, Per, Fin, Lis y Mif. 
4) Se tiene la referencia de la vertical, pero la forma de algunos objetos impide que lleguen a ella. Después del experimento se aprecian bien las diferencias, generalmente con precisión, y se toma conciencia de que la forma no inffuye tanto como se preveía, exceptuando eI aro: Luk, Mer, Gal, Ter y Gon.

5) Desde el primer momento, la bola toma la vertical con independencia de la forma. Después de la experiencia, el sujeto no ve necesario dibujar los objetos de nuevo, ya que el esquema inicial de la vertical se mantiene: Man y Sim.

El alto grado de significación de las respuestas se pone de manifiesto de dos modos diferentes:

1) La diversidad de categorías obtenidas sobre Ias respuestas muestra la riqueza de matices (no se aprecian tendencias dicotómicas tipo «conocer-desconocer»).

2) Se obtienen una gran variedad de respuestas novedosa respecto a los datos perceptivos de las situaciones físicas presentadas.

Se puede comprobar que, si bien el contenido es significativo para la mayoría de los alumnos, no lo es para otros como Pek, Lor, Lil, Van y Ram, las respuestas de los cuales dependen de los aspectos figurativos de tareas presentadas, mientras que para otros sujetos como Des, Fan, Zip, Rev, etc. utilizan verdaderos esquemas, que pueden deducirse de sus explicaciones y que son independientes de los aspectos perceptivos de tareas presentadas (Marín, 1994).

\section{Ejemplo de conceptos de un nivel de significación baja a pesar de las tareas}

En este caso se ha seleccionado el concepto de diferencia de potencial eléctrico (ddpe), el cual consideramos que presenta un bajo nivel de significación, según se deduce del criterio anteriormente planteado y que se tratará de justificar a continuación.

Shipstone (1989), para determinar las ideas, dificultades y tipos de razonamiento que utilizan los alumnos británicos, de edades comprendidas entre 11 y 18 años en la fenomenología eléctrica y, más concretamente, sobre el concepto de voltaje, utilizó un circuito eléctrico cerrado en serie constituido por varias pilas y dos resistencias; una de las cuales es variable. Además, dos voltímetros se conectan en paralelo, el $V_{1}$ en la resistencia fija y el $V_{2}$ en la variable. Se hacía la pregunta siguiente: Si se aumenta el valor de la resistencia variable, $\iota$ qué sucederá con los valores que indican los voltímetros? Realiza una explicación de la decisión que has tomado.

Los resultados que obtuvo para el concepto de voltaje, en alumnos de cuarto que habían estudiado en aquel mismo año los circuitos, fueron los siguientes:

I) El $75 \%$ de los alumnos no se refiere al voltaje, a pesar de haberlo estudiado anteriormente en el mismo año.
Los alumnos explicaban su elección en términos de corriente, electricidad o potencia

2) El $31 \%$ de los alumnos que habla sobre el término de voltaje, lo consideran como algo que fluye, es decir, responde de la siguiente forma: «I a lectura del voltímetro $\left(V_{2}\right)$ aumenta porque pasa más voltaje a través de la corriente en $\mathrm{V}_{2,3}$

3) Los razonamientos que utilizan algunos alumnos son del tipo: «La corriente que pasa por el circuito tratará de tomar el camino más fácil posible, por lo que en vez de pasar por la resistencia variable, irá por eĺ sitio menos difícil,»

Para todas las respuestas, Shiptone detecta que para el alumno existe «algo», aun después de haber estudiado los circuitos, que fluye por los conductores. Para explicar la situación se basan en dos ideas fundamentales, ambas relacionadas con aspectos figurativos de las tareas:

a) el aumento o disminución de los valores de los voltímetros;

b) los conductores del circuito.

En realidad el sujeto se limita a razonar secuencialmente (dirección del fluido en el interior del circuito) y a pasar la corriente por los voltímetros o por la resistencia variable, según le interese para justificar lo observado (aumento o disminución de los valores del voltímetro).

Si se aplica el criterio de significación establecido, encontramos que las respuestas de los alumnos son tomadas de los aspectos figurativos de la tarea y evocan unas veces aspectos irrelevantes, y otras sustituyen en sus explicaciones el concepto de ddpe por el de corriente eléctrica. Por otro lado, la pregunta inicial es bastante cerrada, aunque luego las explicaciones pucieran ser abiertas; así, nos quedaba la duda de si las respuestas dadas por los alumnos eran no significativas por el tipo de preguntas realizadas o por la propia situación f́́sica propuesta.

En un intento de cambiar los aspectos perceptivos de la situación fúsica y con el fin đe ratificar la hipótesis de que el concepto de ddpe tiene poco significado para la mayoría de los alumnos, pasamos un protocolo de entrevistas. Algunas de las situaciones físicas, preguntas y respuestas dadas por un alumno se exponen en el cuadro 1 .

En este cuadro aparecen cinco columnas: en la primera, se presentan las situaciones físicas y las cuestiones iniciales; en la segunda, los resultados que los alumnos observaban o pensaban que iban a ocurrir antes de llevar a cabo la conexión del circuito; en la tercera, el resultado experimental que se observa después de cerrar los circuitos; en la cuarta, las preguntas del entrevistador después de que el alumno ha observado los circuitos una ve\% puestos en funcionamiento; y en la quinta, se exponen algunas de las explicaciones que dieron los alumnos a las preguntas de la columna cuatro. 
Cuadro I

\begin{tabular}{|c|c|c|c|c|}
\hline SITUACION FISICA Y CUESTIONES & $\begin{array}{l}\text { RESULTADO } \\
\text { ESPERADO }\end{array}$ & $\begin{array}{c}\text { RESLLTADO } \\
\text { EXPERIMENTAL }\end{array}$ & PREGUÑTAS & $\begin{array}{l}\text { EXPLICACION } \\
\text { DE LN ALUMNO }\end{array}$ \\
\hline $\begin{array}{l}1^{\circ} \text { ) Se presentan los circuitos siguientes: } \\
\text { Se hace observar al alumno la iuminosidad } \\
\text { de las bombillas. Se aprecia que } \mathrm{B}_{1} \text { ilumina } \\
\text { más que } \mathrm{B}_{2} \text {. }\end{array}$ & & $\begin{array}{l}\text { Ilumina más la } \\
\text { bombilla } B_{1}\end{array}$ & $\begin{array}{l}\text { ¿Podrias dar } \\
\text { alguna explicación } \\
\text { de por qué ilumina } \\
\text { más la bombilla } B_{1} \text { ? }\end{array}$ & $\begin{array}{l}\text { Porgute le flega } \\
\text { más energía. }\end{array}$ \\
\hline $\begin{array}{l}\text { 29) Ahora se presenta el circuito de este } \\
\text { modo: } \\
\text { Antes de poner en funcionamiento el cir- } \\
\text { cuito, se pide a alumno que prediga qué } \\
\text { bombilla iluminará más cuando se cierra } \\
\text { ef circuito } \mathrm{C} \text {. }\end{array}$ & $\begin{array}{l}\text { Respuesta del } \\
\text { alumno: } \\
\text { iluminará más } \\
\text { la } B_{1} \text { porque le } \\
\text { llega más } \\
\text { energía. }\end{array}$ & $\begin{array}{l}\text { Al realizar las cone- } \\
\text { xiones oportunas } \\
\text { del circuito C, se } \\
\text { observa que lace } \\
\text { más la bombilia } \mathrm{B} \text {. }\end{array}$ & $\begin{array}{l}\text { ¿Podrías dar alguna } \\
\text { explicación al fenó- } \\
\text { meno observado? }\end{array}$ & 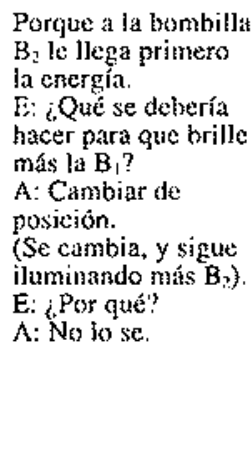 \\
\hline $\begin{array}{l}\left.3^{\circ}\right) \text { Finalmente se dispone así: } \\
\text { Antes de cerrar cl circuito, se pregunta al } \\
\text { entrevistado que prediga qué bombilla se } \\
\text { iluminará más. }\end{array}$ & $\begin{array}{l}\text { A: Se iluminará } \\
\text { más la } B_{1} \\
\text { E: ¿Por qué? } \\
\text { A: Porque le } \\
\text { llega más } \\
\text { energía. }\end{array}$ & $\begin{array}{l}\text { Se ilumina más la } \\
\text { bombilia } B_{1} \text {. }\end{array}$ & $\begin{array}{l}\text { E: ¿Por qué se } \\
\text { ilumina más la } \\
\text { bombilla } \mathrm{B} \text { : que } \\
\text { la } \mathrm{B}_{2} \text { ? }\end{array}$ & $\begin{array}{l}\text { A: Porque le llega } \\
\text { más energía. }\end{array}$ \\
\hline
\end{tabular}

Mediante una entrevista individual, se pasaron las situaciones físicas y las preguntas del cuadro 1 a un grupo de 20 alumnos comprendidos entre 12 y 22 años de edad, procedentes del ciclo superior de EGB, bachillerato y de la Escuela Universitaria de Magisterio. Los resultados obtenidos fueron:

1) Los alumnos de 12 años explican la iluminación de la bombilla por Ia energía que le llega. Si se ilumina más, es porque le llega más y, por el contrario, menos porque le Ilega menos. Iguales explicaciones suelen utilizar los alumnos de primer ciclo de universidad (Escuela Uni- versitaria de Magisterio, especialidad de Ciencias Físico-naturales).

Lo anterior puede explicarse porque frases como apaga la luz o desconecta el televisor, que gasta mucho inducen a los sujetos a pensar que de la pila o enchufe de la vivienda sale energía, que se pierde o se gasta en la bombilla o el televisor.

2) Se ha podido observar que alumnos de la Escuela Universitaria de Magisterio, igual que los de 12-16 años, encierran bajo el término electricidad los conceptos de 
intensidad, ddpe y fem. Los términos más utilizados fueron: energía, conductores, circuito, bombilla, pila, voltios para caracterizar la pila, polo positivo y negativo, que coinciden, como es lógico, con las palabras que describen los objetos que se utilizan en la vida diaria.

Cuando utilizan el término de voltio es para referirse a la capacidad de la pila, potencia de la bombilla o potencia de la pila. El voltaje sólo es utilizado a la hora de diferenciar bombillas o diferenciar pilas.

3) Ningún alumno utilizó el concepto de ddpe para explicar la diferencia de luminosidad de las bombillas, a pesar de que algunos de ellos habían estudiado electricidad previamente.

4) La mayoría de los alumnos entrevistados utilizó un razonamiento secuencial. Así, piensan que al cambiar las bombillas cambiarfa su luminosidad. Al realizar la experiencia y observar que no se producían cambios, quedaban sin respuesta para justificar la nueva situación experimental.

Lo anterior puede justificar el escaso significado que tiene el concepto de ddpe para los alumnos entrevistados, hecho que puede ser explicado porque la fenomenología eléctrica sólo se observa à través de sus efectos. De aquí, que el sujeto no tenga estímulos, percepciones, interacciones suficientes que originen el desarrollo cognitivo correspondiente a dicha fenomenología (Jiméne $z$ Gómez y Fernández Durán, en prensa).

Lo máximo que se podría decir sobre el concepto de ddpe es que constituye un conocimiento ingenuo o de sentido común generado por interacción con el mundo físico y social, a través de lo que Farr y Moscovici (Pozo et al., 1991) han llamado representaciones sociales, pero que su significado dista mucho de lo que sería un contenido significativo.

\section{SUGERENCIAS METODOLÓGICAS}

El problema que nos ocupa no sólo reside en la significación del contenido que queremos investigar, sino también en el modo de obtener información del alumno. Así, si se hacen preguntas, verdaderas o falsas, o de opción múltiple o bien si se enfatiza sobre el contenido académico, aumentan las posibilidades de que las respuestas obtenidas sean de compromiso. De esta manera se evidencia más el desconocimiento que tienen los sujetos sobre el contenido objeto de investigación que lo que realmente interesa: $a$ ) la obtención de información acerca de los esquemas explicativos e ideas que usan los alumnos para explicar la situación física planteada; b) los esquemas cognoscitivos que utiliza para hacer previsioncs, interpretaciones o explicaciones de dichos fenómenos.

Lo anterior nos ha llevado a considerar la entrevista individual como técnica que utiliza un acercamiento progresivo al conocimiento del entrevistado, evitando sesgos y distorsiones, ya que al construir buena parte de las preguntas sobre la base de sus respuestas, se enfatiza el punto de vista del alumno. Las pautas que se suelen seguir son fundamentalmente las siguientes:

a) Elección del contenido objeto de enseñanza que se quiera investigar y selección o diseño de un protocolo de entrevistas (situaciones físicas y preguntas) donde subyace dicho contenido y que permite obtener información significativa del sujeto de interés didáctico (Marín, 1994).

b) Depuración del cuestionario; para ello se utiliza el criterio de significación a través de las reacciones de los encuestados (expresiones verbales, gráficas y manipulaciones del material). Es necesario:

- Diversificar los individuos de la muestra.

- Modificar los elementos constituyentes y diferenciables del cuestionario: preguntas iniciales, dinámica de la entrevista, formas de presentar la situación física y la manipulación del material.

c) Control de la influencia de los aspectos perceptivos de la situación física planteada. Esto nos permite:

- Analizar la capacidad de los sujetos para responder por encima del engaño perceptivo.

- Evaluar el grado de flexibilidad o estabilidad de los datos empíricos.

d) Si a través de las respuestas de los alumnos entrevistados, después de la depuración del cuestionario, se siguen teniendo reacciones del sujeto poco significativas, se podrá decir que el nivel đe significación del contenido investigado será bajo o nulo para el sujeto o sujetos entrevistados. De esta manera se podrá delimitar el contenido significativo del que no lo es.

Las respuestas de los sujetos cuando provienen o son reflejo de la activación de uno o varios esquemas cognoscitivos deben presentar cierto grado de estabilidad ante variaciones perceptivas y estructurales de los factores que intervicnen en lat tarea (Marín, 1995). Por esto, es conveniente analizar si ante condiciones semejantes se obtienen respuestas del sujeto análogas (repetición) y si las respuestas perduran ante cambios de situaciones físicas (generalización).

Una metodología que no ponga en juego variaciones perceptivas y estructurales de los factores que intervienen en la tarea impide analizar el grado de estabilidad de las respuesta de los sujetos.

Cuando los alumnos dan respuestas basadas en aspectos perceptivos de la situación física presentada o bien son de compromiso, consideramos que éstas no son reflejo de un determinado esquema cognoscitivo. En tal caso, si el investigador las interpreta como si fuesen auténticas concepciones, catalogándolas de «errores conceptuaIes», está suponiendo que el sujeto tiene experiencia 
relativa al concepto investigado, cuando en realidad no la tiene. Además, si se hacen diseños de enseñanza basados en dichas "concepciones", los resultados podrían ser poco consistentes.

Para sintetizar lo anterior diríamos que:

1. Fl contenido objeto de enseñanza puede clasificarse por su nivel de significación para un determinado alumno, según que haya generado o no esquemas a través de sus interacciones.

2. Sólo se puede saber si un contenido tiene o no significado para un alumno cuando se analizan las repuestas de éste a preguntas realizadas sobre situaciones físicas donde se involucra dicho contenido.

3. No se debe identificar y, menos aún, describir concepciones para aquellos contenidos que no tienen significado para los alumnos.

\section{REFERENCIAS BIBLIOGRÁFXCAS}

ACEVEDO, J.A. (1990). Aportaciones acerca del aprendizaje por analogía: modelos analógicos y conceptuales de la corriente eléctrica. Cambioeducativo y desarollo profesional. Actas de las VII Jornadas de Fstudio sobre la Investigación er la Escuela, Sevilla, pp. 201-208.

CARMICHAEL, $P$. et al. (1990). Rechearh on students' conceptions in science: a bibliography. Children's learning in Science: Unjversity of Leeds

CLOSSET, J.L. (1989). Les obstacles a l'apprentissage de l'electrocinétique. Bulletin de L'Union des Physciens, 716, pp. 931-949.

CONFRFY, I. (1990). A Review of the Research on Student Conceptions in Mathematics, Science and Programming, pp. 3-56, en Cazuen, C. (eds). American Education Research Association. Michigan State University: Review of Educational Research.

GABFI,L, D., SAMUEL, K y JUNN, D. (1987). Understanding the particulate nature of matter. Journal of Chemical Education, 64(8), pp. 695-697.

HHILER, P.M. y FIN LEY, F.N. (1992). Variable Uses of Alternative Conceptions: A Case Study in Current Electricity. Journal of Research in Science Teaching, Vol. 29(3), pp. 259-275.

HIERREZUELO, J y MONTERO, A. (1989). La ciencia de los alumnos. Barcelona: Laia-MEC.

MARÍN, N. (1994). Evolución de los esquemas explicativos en situaciones de equilibriomecánico. Tesis doctoral. Universidad de Granada.

MARÍN, N. (1995). Metodología para obtener información del alumno de interés didáctico. Almería: Servicio de Publicaciones de la Universidad de Almería.

JIMÉNEZ GÓMEZ, E., SOLANO, 1. y MARÍN, N. (1994). Problemas de terminología en estudios realizados sobre «lo
4. Los diseños de enseñanza que se realicen para el aprendizaje de conceptos requieren de estrategias diferentes, según sea o no significativo para el alumno.

5. La técnica de enseñanza basada en el cambio conceptual sólo debería ser aplicada a aquellos contenidos de un alto nivel de significación, puesto que los alumnos han generado esquemas sobre ellos (diferentes a los que tiene el profesor) y son dichos esquemas precisamente los que se pretenden modificar.

\section{NOTA}

El presente trabajo ha sido financiado por la DGICYT como parte del proyecto PS93-0174 del Programa Sectorial de I'romoción General del Conocimiento.

que el alumno sabe» en ciencias. Enseñanza de las Ciencias, Vol. 12(2), pp. 235-245.

JIMÉNEZ GÓMEZ, E. y FERNÁNDFZ, DURÁN, E. Didactic Problems in the Concept of Electric Potential Difference and an Analysis of its Philogenesis. Science and Education. (En prensa)

MARÍN, N. y JIMENEZ GOOMEZ, E. (1995). Contenidos académicos y contenidos delalumno, pp. 125-128, en Calonge, A. Actas de los XIII Encuentros de Didáctica de las Ciencias Experimentales. Guadalajara: Servicio de Publicaciones de la Universidad de Alcalá de Henares.

MCCLELLAND, J.A. (1984). Alternative frameworks: interpretation of evidence. Eur. J. Sci. Educ., Vol. 6(1), pp. $1-6$.

PIAGET, J. (1977). Epistemología Genética. (Solpín: Argentina). Traducido de L'epistemologiegenitique. (1974). (París: Presses Universitaires de France).

PIAGET, J. (1981). Psicologia y Epistemología. (Barcelona: Ariel). Traducido de Psychologie et épistémologic. (1970). (París: Denoël-Gonthier).

PIAGET, J. y GARCÍA, R. (1973). Las explicaciones causales. Barcelona: Barral.

PO7O, J.I., SANZ, A., GÓMEZ, M.A. y LIMÓN, M. (1991). Las ideas de los alumnos sobre ciencia: una interpretación desde la psicología cognitiva. Enseñanza de las Ciencias, Vol. 9(1), pp. 83-94.

SHIPSTONE, D. (1989). Electricidad en circuitos sencillos, en Driver, R., Guesne, E. y Tiberghein. Ideas cientificas en la infancia y la adolescencia. Madrid: Morata.

WATTS, D.M. y 7,YLBERSTTAJN, A. (1981). A survey of some children's ideas about force. Physics Education, Vol. 16. pp. $360-365$. 